\title{
A systematic review of smartphone apps for gastro-oesophageal reflux disease: the need for regulation and medical professional involvement
}

\author{
Lekshmi Suseela Venugopal $^{1 \wedge}$, Aya Musbahi ${ }^{2}$, Venkatesh Shanmugam ${ }^{1}$, Bussa Gopinath ${ }^{2}$ \\ ${ }^{1}$ Department of Colorectal Surgery, University Hospital of North Tees, North Tees and Hartlepool NHS Foundation Trust, UK; ${ }^{2}$ Department of \\ Upper GI Surgery, University Hospital of North Tees, North Tees and Hartlepool NHS Foundation Trust, UK \\ Contributions: (I) Conception and design: A Musbahi; (II) Administrative support: All authors; (III) Provision of study materials or patients: A \\ Musbahi; (IV) Collection and assembly of data: LS Venugopal; (V) Data analysis and interpretation: LS Venugopal; (VI) Manuscript writing: All \\ authors; (VII) Final approval of manuscript: All authors. \\ Correspondence to: Lekshmi Suseela Venugopal. Foundation Year 2, King's Mill Hospital, Sutton-in-Ashfield, UK. Email: lekshmi.venugopal@nhs.net.
}

Background: Expansion in smartphone use and mobile health (mHealth) apps has generated a large and
unregulated sector. Gastro-oesophageal Reflux Disease (GORD) is a widely prevalent disease in the UK. The
aim of this study is to evaluate the smartphone apps focused on GORD, available on major digital platforms,
with particular emphasis on their recorded evidence base (EB) and the extent of medical professional
involvement (MPI) in their constitution.

Methods: Relevant apps were extracted using defined search terms and inclusion (using a wide array of search terms) and exclusion criteria (non-English language, duplicates) were applied. Data was collected from the overview provided by the developer in the app store and from the developer website for each app, and classified according to various variables. EB and professional involvement data was obtained from the application details provided in the app stores as well as information section of individual apps.

Results: A vast majority of the apps (97.3\%) were developed targeting the general public. Of the 73 apps, $11(15.1 \%)$ had a documented EB. Two apps stated if healthcare professionals were involved in the development, screening or assessment of the app content, meaning only $2.73 \%$ of the apps (2/73) stated their content had been contributed by medical professionals (individual, group or organisation of health providers). Thirty-four apps had recent updates in 2019 (47\%, 34/73).

Conclusions: Regulation and accreditation of mHealth apps related to GORD are needed.

Keywords: Smartphone applications; mobile health (mHealth); Gastro-oesophageal Reflux Disease (GORD)

Received: 10 June 2020; Accepted: 31 January 2021; Published: 20 October 2021.

doi: $10.21037 /$ mhealth-20-126

View this article at: http://dx.doi.org/10.21037/mhealth-20-126

\section{Introduction}

The first handheld mobile phone came into existence in 1973 , weighing $1.1 \mathrm{~kg}$, allowing 30 contacts, a talk time of 30 minutes and 10 hours of recharging time $(1,2)$. In the world of the millennials, smartphones are a technological phenomenon, considered quite indispensable by modern society.

The recent statistics have estimated the number of mobile phone users (smartphones and feature phones) in 2020, is 4.78 billion, which makes $61.62 \%$ of the world's population a cell phone owner. As per Statista, the number of smartphone

\footnotetext{
$\wedge$ ORCID: 0000-0002-0128-6413.
} 
users in the world currently is 3.5 billion, which means $45.12 \%$ of the population use a smartphone. This is a considerable rise from 2016 when there were only 2.5 billion users, $33.58 \%$ of that year's global population $(3,4)$.

Smartphones are multifunctional mobile computing devices with robust hardwares and efficient operating systems incorporating a wide range of multimedia components-music, videos and games, digital photography and dynamic mobile internet facilities, alongside the primary phone functions such as voice calls and text messaging.

Smartphone apps are available to download from application distribution platforms operated by the owner of the mobile operating system, such as the App Store and Google Play Store (for iPhones and Android phones). Some apps are free, and others have to be paid for, and the profit will be shared between the creator of the application and the distributor.

Mobile health (mHealth) is defined as the delivery of healthcare or health-related services through the use of portable devices. It is estimated that hundreds of applications are added each day on the major platforms and presently around 318,000 health apps are available worldwide, almost double to what was available two years ago. Around 204 billion apps were downloaded in 2019 (up from 140.7 billion app downloads in 2016), generating revenues of over 462 billion USD. According to Statista, by 2023, mobile apps are projected to generate more than 935 billion U.S. dollars in revenues via paid downloads and in-app advertising (5).

Majority of the mHealth apps available are for general wellness. However, health condition management apps, i.e., those associated with patient care are increasing at an exponential rate, now representing about 40 per cent of all health-related apps. There are 571 published studies aimed at the essence of app efficacy with the availability of ample clinical evidence, which has enabled the identification of a list of top apps with increasingly robust clinical evidence (6). Medical apps in widespread use by healthcare professionals currently include Epocrates, PEPID and Medscape apps which are clinical reference tools, and the MedCalc app which is widely used in the UK that provides point-of-care clinical decision/support tools like various medical scoring systems and calculators, and diagnostic and management algorithms.

The previous studies on evaluating the use of mHealth apps has been targeted on obesity management $(7,8)$, obesity surgery (9), various branches of surgery (10-13), cardiovascular disease (14) and a few others (15-19). These papers have highlighted concerns regarding misleading substance, scarce evidence-based content and the exigency of more medical professional involvement (MPI).

Gastroesophageal reflux disease (GORD) is a common global disease with increasing prevalence and consequently greater burden on the healthcare system, and despite this, little research has been done to evaluate GORD mHealth applications. The UK prevalence of dyspepsia ranges from $12 \%$ to $41 \%$. It is estimated that annually around $40 \%$ of the UK adult population experience dyspepsia (20).

The NHS has a website that lists mApps and other online tools, (https://www.nhs.uk/apps-library/), which can be accessed by the public to choose quality applications that are evidence-based and have MPI. Searching the NHS library has given no results concerning GORD related apps.

This study is a systematic review of the smartphone apps focused on GORD, available on major digital platforms, with particular emphasis on their recorded evidence base (EB) and the extent of MPI in their constitution, classify it as per the NICE Evidence Standards Framework (21) and analyse what is lacking in quality standards compared to the NHS validated apps and apply a scoring system, the Silberg score to these apps (22).

We present the following article in accordance with the PRISMA reporting checklist (available at http://dx.doi. org/10.21037/mhealth-20-126).

\section{Methods}

Smartphone applications focusing on Gastroesophageal reflux disease were identified by searching the four largest digital platforms (Apple App Store, Google Play, BlackBerry World, Windows Phone). A wide array of search terms were used to include all possible applications available to download with British English and American English spellings. Search terms used were heartburn, dyspepsia, acid reflux, gastric reflux, acidity, indigestion, regurgitation, fundoplication, anti-reflux, antireflux surgery, anti-acid, GORD, GERD, gastro-oesophageal reflux, gastro-oesophageal reflux, antacid, water brash, gastritis, duodenitis, oesophagitis, esophagitis.

App data was collected from the description detailed by the developer in the app store and from the developer website, and classified according to various variables: (I) Platform, (II) year of release and last updated date, (III) developer and Name of the Application, (IV) App accessibility, (V) commercial interests (if any), (VI) App functionality and App store Category, (VII) documentation 
of MPI, (VIII) documentation of EB, (IX) potential safety concerns, $(\mathrm{X})$ number and length of written reviews, and (XI) Number and score of star rating reviews.

The relevant applications available on Google Play and Apple App store ${ }^{\circledR}$ were listed for inclusion. The study sample consisted of applications that were intended for the general public including patients, families and carers, and healthcare professionals. Only English language applications specifically related to GORD were included in the study sample. Therefore apps that integrated GORD as a small component among a vast range of topics were excluded as their primary focus was not GORD. Both free apps and paid apps were included. Foreign language apps, apps related to peptic ulcer and ulcer diet and apps targeted at alkaline diet were excluded.

\section{Data collection and analysis}

Details from app store descriptions were extracted from the app marketplaces - Google Play, Apple App Store and Windows Phone Store, from 01 December 2019 to 31 December 2019. Information was collected on the following variables: app name, platform (Apple App Store, Google Play or Windows), developer affiliation, cost, date of last update, and interactive features.

All the data were collected online and in the public domain, and was recorded on a Microsoft Excel spreadsheet for collation and analysis by two separate authors LV and AM. Apps were analysed according to their purpose into the following categories. This was in keeping with a similar study by Mobasheri et al., which also classified apps in breast surgery according to these tools (12):

(I) Education \& Information tools: general information and tools to raise public recognition of GORD symptoms; includes descriptions, causes and treatment options.

(II) Dietary tools: tools to attract the general public by providing recipes and lists of food to either include or exclude in their daily diet plan.

(III) Patient diary tools: Logbook, calendar and notepad for the patients to track medication usage, symptoms, to schedule appointments etc. to be used as their personal health record.

(IV) Audiovisual tools: chiefly aimed at medical professionals, giving video lectures and animated videos.

(V) Social networking tools: Providing the users with interactive features option to communicate with each other and share their experiences, access to peer assistance, includes information/photo sharing.

(VI) Self-assessment tools: information to assist in the identification of GORD symptoms, and information and practical tools to deal with the medical, behavioural, or emotional aspects of GORD.

The classification system that we adapted is not standardized. We modified the existing categories in android and apple stores to aptly describe the various mHealth apps categories. However, we recognized the importance of adding a standardized classification to analyze to obtain results that are not arbitrary, hence we added the Evidence Standards Framework for Digital Health Technologies (DHTs) (21) developed by NICE between June 2018 and February 2019 in collaboration with NHS England, Public Health England and MedCity.

It has been mentioned that the framework was designed for DHTs that are commissioned in the UK health and care system and it is less relevant to the DHTs that are downloaded or purchased directly by users from app stores. However, analyzing the applications based on the tier system was necessary to truly ascertain the quality of the mHealth applications available in mobile app stores.

The following are the tier classifications

(I) Tier 1: DHTs with potential system benefits but no direct user benefits.

(II) Tier 2: DHTs which help users to understand healthy living and illnesses but are unlikely to have measurable user outcomes.

(III) Tier 3a: DHTs for preventing and managing diseases. They may be used alongside treatment and will likely have measurable user benefits.

(IV) Tier 3b: DHTs with measurable user benefits, including tools used for treatment and diagnosis, as well as those influencing clinical management through active monitoring or calculation. It is possible DHTs in this tier will qualify as medical devices.

The evidence tiers are cumulative and it is clearly stated that the DHT must meet all the standards in the previous tier(s), as well as its own tier. This limited our classification to a great extent, for example, some applications exclusively provided tracking tools but no information or a 2-way communication between the user and the professional, which meant that we could not assign such apps Tier 3 a but only Tier 2. However, since sending the data is optional and 


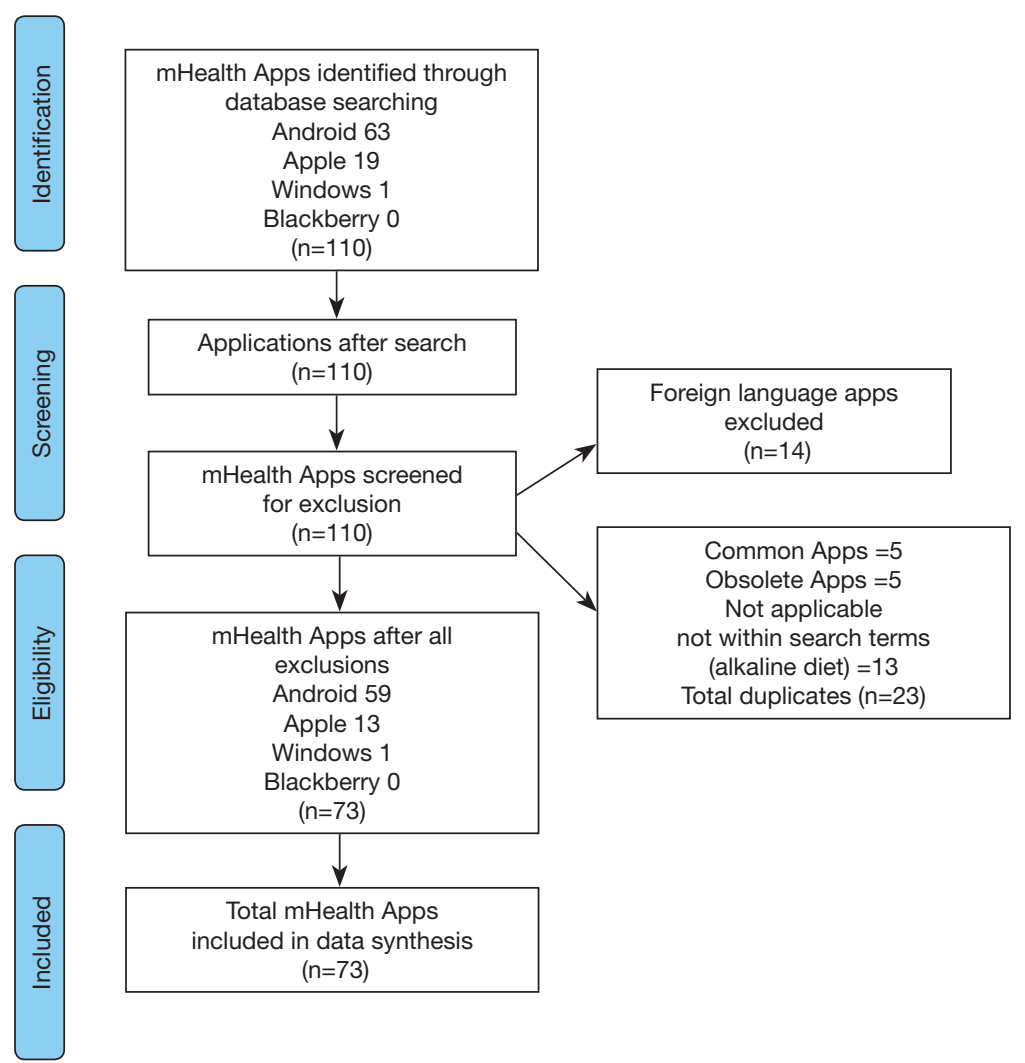

Figure 1 PRISMA flow chart (n=number of applications).

to enhance our study results, we have assigned Tier 3 a to the applications that provide symptom tracking functions. Furthermore, no application could be classified as Tier 1, since they are applications to improve system efficiency and do not have any direct and measurable individual patient outcomes.

The established six categories were applied to all 73 apps in the study and classified according to their primary function and tier classification. A few apps included more than one function, and all the functions that the app provided were included in the spreadsheet. Any differences in the groupings of the apps into one of the six categories were resolved by the two researchers until an agreement was achieved.

\section{Silberg score}

The Silberg scoring system was applied to all the identified apps and a score of 9 maximum could be achieved. The score is a validated tool for looking at the quality of healthcare information digitally. No peer-reviewed scoring systems exist especially for mHealth apps but a modified Silberg score has been used for bariatric surgery and depression mHealth apps.

\section{Results}

The assorted keywords were used for the initial search in Google Play, iOS App Store and Windows store individually, and an initial 110 potential apps were identified (Google Play =63; Apple Appstore $=19$; Windows store $=$ 1 ; Blackberry world $=0$ ). Out of these, 13 alkaline diet apps and 14 foreign language apps were removed. Five apps were identified as duplicate versions with the same name and developer in 2 or more platforms. The five duplicates were removed from the less popular app market, hence 5 apps were excluded from the Apple App Store. The remaining 78 apps were individually downloaded for further screening, and 5 apps were found to be obsolete, which were excluded. A total of 73 apps met all the inclusion criteria and were included in our final study sample (Google Play =59; Apple Appstore $=13$; Windows store $=1 ;$ Figure 1 ). 

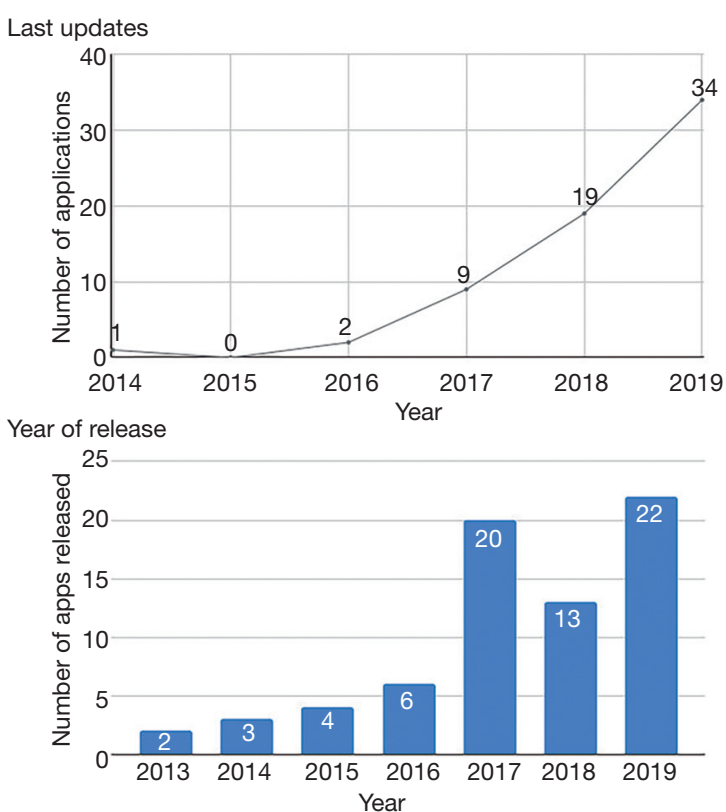

Figure 2 Charts showing last updates of GORD Apps and number of GORD Apps released between 2013 and 2019. GORD, Gastrooesophageal Reflux Disease.

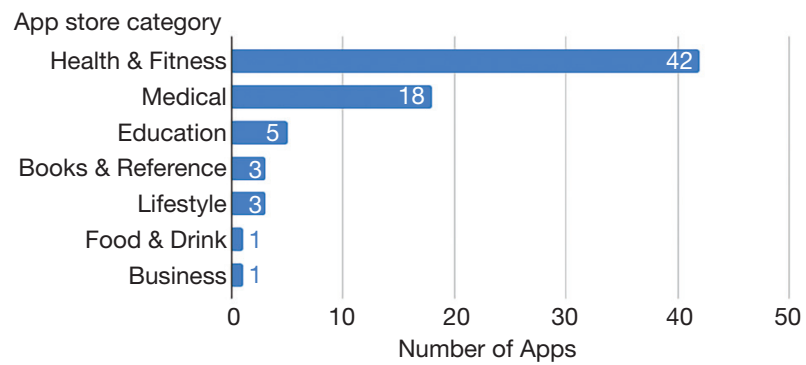

Figure 3 Chart showing the number of GORD apps in various categories. GORD, Gastro-oesophageal Reflux Disease.

Table 1 GORD App functions ( $\mathrm{n}=$ number of applications)

\begin{tabular}{lcc}
\hline Category & $\mathrm{n}$ apps & Percentage \\
\hline Education \& Information tools & 36 & $49 \%$ \\
Dietary tools & 44 & $60 \%$ \\
Patient diary tools & 8 & $11 \%$ \\
Audiovisual tools & 2 & $3 \%$ \\
Social networking tools & 1 & $1 \%$ \\
Self assessment tools & 1 & $1 \%$ \\
\hline
\end{tabular}

GORD, Gastro-oesophageal Reflux Disease.

\section{App availability}

Of all the available applications, most were free to download (79.45\%, 58/73), five apps offered In-App purchases (6.84\%, $5 / 73)$ and ten $(13.69 \%, 10 / 73)$ required payment (Figure 2). The Google Play store had 56 apps available free of charge and three had costs ranging from $£ 0.99$ to $£ 4.59$, while Apple app store provided 6 apps for free and seven apps required payment (£1.99-£4.99).

\section{Date of last update}

Out of the 73 apps in the study group, the earliest releases were in the year 2013. Two apps were released that year, and thereafter a general increment every following year, except in the year 2018. Twenty apps were released in 2017, 13 apps in 2018, and 22 apps in 2019.

The study examined the apps that had been updated over a 5 -year period. Thirty-four apps had recent updates in $2019(47 \%, 34 / 73)$ and nineteen apps were last updated in $2018(26 \%, 19 / 73)$, and nine had their last update in 2017 (12\%, 9/73). Four apps did not have any details regarding their recent updates, all of them from the Apple store (5\%, 4/73; Figure 2).

\section{App store categories and functions}

The app marketplaces classified the 73 applications into various categories; $57.5 \%$ were categorised as Health \& Fitness, $24.7 \%$ as Medical, $6.8 \%$ as Education, $4.1 \%$ each in the Books \& Reference, and Lifestyle categories, $1.4 \%$ each in Food \& Drink and Business categories (Figure 3).

The primary functions of the applications targeting GORD were summarised using six key categories. Some of the applications included in our study population had more than one functionality, and all applicable categories were assigned to each app. According to the classification design, dietary apps were the most predominant, comprising $60 \%$ of the apps in the study sample $(60 \%, 44 / 73)$. This was followed by educational/informational apps (49\%, 36/73). Patient record/ diary management apps constituted $11 \%$ of the apps $(11 \%, 8 / 73)$. This was followed by apps for audio-visual enabled studies (3\%, 2/73), Social networking apps $(1 \%, 1 / 73)$ and self-assessment apps $(1 \%, 1 / 73)$. Table 1 shows the primary app functions targeted by the applications in our study sample. 


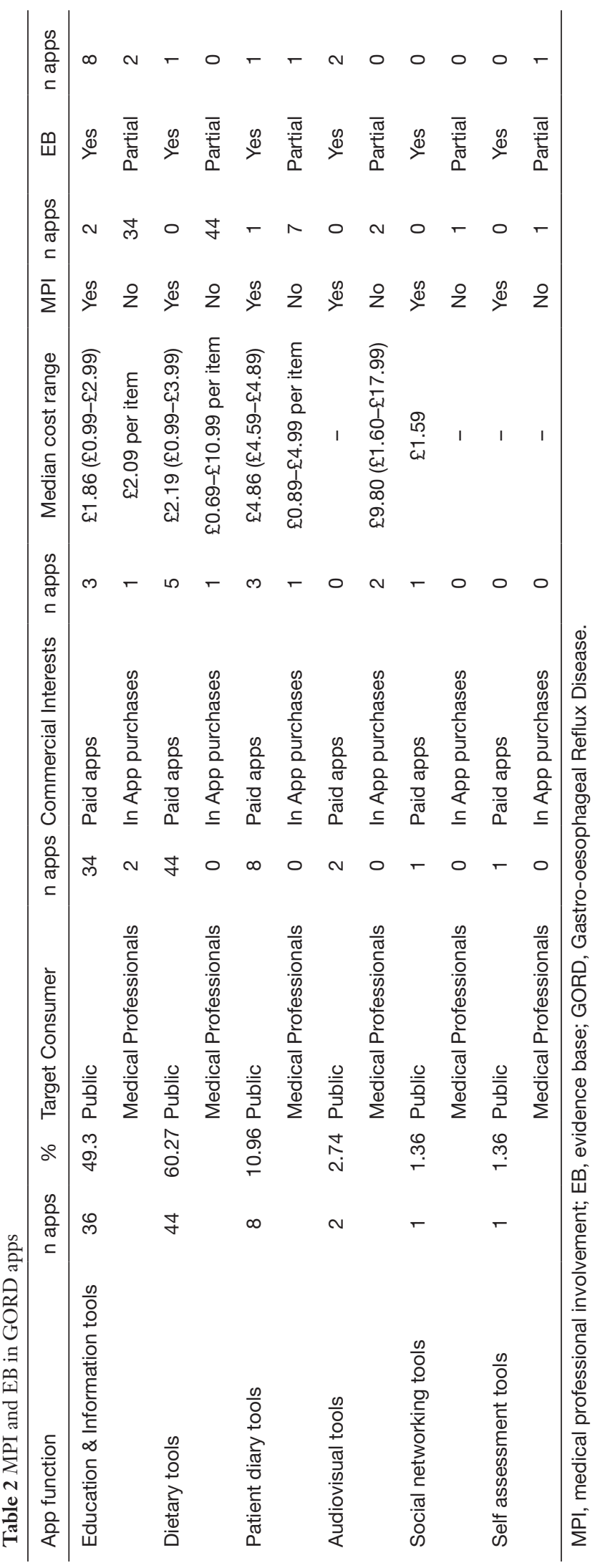

Sixty percent of the apps (44/73) asserted to provide advice regarding "acid reflux preventing food" and nutrition, including home remedies and alternative medicine. They also provided a glossary of food items and recipes for mobile users.

\section{Target consumers}

A vast majority of apps, 71 (97.3\%), were developed targeting the general public. Only 2 apps $(2.7 \%)$ were targeted at healthcare professionals (including medical students/doctors/ nurses/allied health care professionals and students).

\section{EB and MPI involvement}

Of the 73 apps, 11 (15.1\%) had a documented EB. Two apps stated if healthcare professionals were involved in the development, screening or assessing the app content; meaning only $2.73 \%$ of the apps (2/73) stated their content had been contributed by medical professionals (individual, group or organisation of health providers), however neither of these specifically named the medical professional(s) involved.

It was also noted that out of the 36 education \& information category apps, only $2(5.6 \%, 2 / 36)$ had a recorded MPI, and $8(22.2 \%, 8 / 36)$ had a documented EB. The sole self-assessment app had no recorded EB or MPI. Table 2 shows the number of applications in each category having MPI and EB, along with their commercial interests in the market.

\section{Evidence standards}

Among the 73 apps, since no applications were for improving the system efficiency, none were assigned Tier 1.63 applications (86.3\%) were classified as Tier 2, 8 applications (11\%) were Tier $3 \mathrm{a}$ and the two applications primarily aimed at medical professionals were not classified under any Tier (Figure 4). The classification of tier 3a could be arguable, since we have assigned that to the apps that offered symptom tracking and managing and the option for the patient to seek medical attention though not directly linked with the app. Only one among the eight applications had MPI in its making, which was also the only app that was evidence based. However, the applications that are available to the users through the NHS apps library are of a superior quality when it comes to symptom tracking and two way communication. One of the apps among the 73 provided 

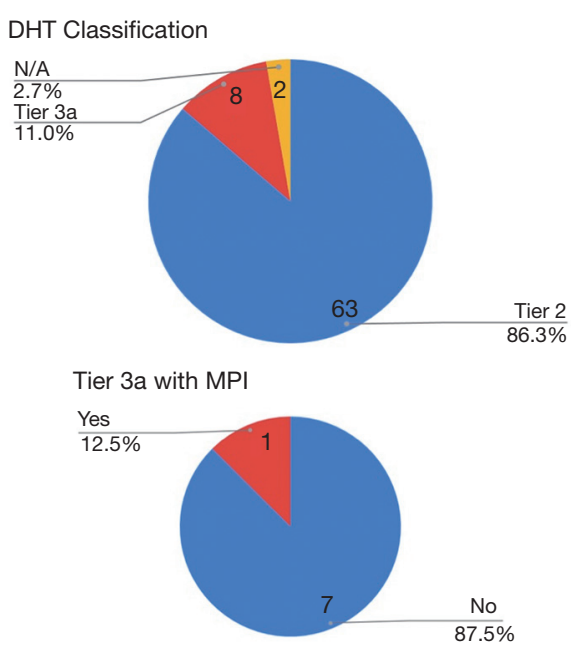

Figure 4 Pie chart showing the evidence standards classification of DHTs and MPI in Tier 3a applications. DHT, Digital Health Technology; MPI, medical professional involvement.

Table 3 Silberg variables and percentages

\begin{tabular}{lcc}
\hline Silberg variables & Percentage of apps & Mean score \\
\hline Authors credited & 10.96 & 0.11 \\
Author affiliations & 2.74 & 0.055 \\
Author credentials & 2.74 & 0.055 \\
Information sources & 19.18 & 0.192 \\
$\begin{array}{lc}\text { Provision of references } \\
\text { Disclosure of application }\end{array}$ & 13.7 & 0.274 \\
ownership & 79.45 & 0.795 \\
Disclosure of sponsorship & 5.48 & 0.11 \\
$\begin{array}{l}\text { Last modification in the } \\
\text { past month }\end{array}$ & 12.33 & 0.123 \\
$\begin{array}{l}\text { Disclosure of date of last } \\
\text { modification }\end{array}$ & 94.52 & 1.89 \\
\hline
\end{tabular}

two way communication among peers, and none offered a connection to a medical professional.

\section{Silberg scoring}

The average Silberg score of the 73 applications in the study was 3.26 \pm 1.29 (maximum score of 9). Only eight applications had a score greater than or equal to that of the mean score. The average scoring for each criteria were as follows: Authorship $0.19 \pm 0.62$, Attribution $0.33 \pm 0.71$,
Disclosure $0.85 \pm 0.49$ and Currency $1.89 \pm 0.46$.

Most of the applications in the study (89.04\%) have not credited the authors. 58 applications have an appropriate disclosure of application ownership (79.45\%), and only nine applications have been modified in the past month/ same month as the data gathering $(12.32 \%)$.

The gap of information quality is prominent in all variables, including the lack of proper author credentials or affiliations (10.96\%), citing the references $(19.18 \%)$ and disclosure of sponsorship (5.48\%). The two variables that scored the highest include the disclosure of the application ownership, and the date of last modification. Table 3 summarizes the average score for each criteria.

\section{Potential safety concerns}

One of the safety concerns that we looked into was the storing of confidential data within the apps. A total of 9 applications $(12.3 \%)$ had the provision to store confidential information in certain forms, including findings from self-assessment, the personal details of the patients including their test results. Only one among the 9 stated that the data will be stored to the user's personal device and no one else will have access to the personal data stored within the app. No other applications mentioned any security measures like passwords or data encryption to protect the confidential data within the app.

\section{Reviews and ratings}

For the consumers to give ratings on the apps, the app marketplaces provide two methods-a star scoring system where an app is given scores from 1-5 stars (worst to best), and a comment section to post written reviews. The users are usually prompted to rate or review the apps while they use it, and the consumers have the option to give a review depending on their level of satisfaction.

Only ten applications among the 73 (13.7\%) had one or more star ratings. The star rating, and number of ratings received are: 4.0 [122], 2.4 [5], 4.7 [47], 4.8 [8], 3.7 [10], 3.7 [12], 3.4 [5], 3.2 [5], 3.5 [2], 5 [1]. The remaining 63 apps had no ratings.

Six apps $(8.21 \%)$ had one or more written reviews. The average length of reviews were 15 words (range, 192 words) and the average number of comments were 18 . Table 4 shows the number of comments and the average word count in the six applications. 
Table 4 Number of comments and average word count in the recorded six applications

\begin{tabular}{lcc}
\hline & Comments & Average words \\
\hline App 1 & 57 & 13 \\
App 2 & 3 & 17 \\
App 3 & 35 & 20 \\
App 4 & 4 & 7 \\
App 5 & 6 & 5 \\
App 6 & 5 & 29 \\
Mean & 18 & 15 \\
\hline
\end{tabular}

\section{Discussion}

Since the definition of mHealth and the generation of more advanced smartphone technology, interest and downloads of mHealth Apps have exponentially increased. Benefits have been shown in patient monitoring and disease control in certain long term conditions such as diabetes, hypertension, obesity, mental health issues and asthma (8,16-19). Buijink et al., 2013, discovered that most mHealth apps that were targeting healthcare professionals lacked clear references, updated policies and contemporary information (23). The study went further in recommending standardisation and regulation to prevent adverse events resulting in a patient safety issue. Moreover, studies that have not been limited to mHealth Apps for health professionals, have also found lack of MPI or a good EB and called for regulation in a systematic review of mHealth apps in musculoskeletal disorders and rheumatoid arthritis by Najm et al., 2019 (24). Reviews of hernia surgery apps and Breast surgery apps also showed similar findings and made similar recommendations $(12,13)$.

The authors present here a review of GORD mHealth applications from an analytical perspective and scoring using the Silberg score which we believe is the first study to be done using this methodology. The authors have found there to be a wide heterogeneity in the calibre and quality of mHealth apps related to GORD. In addition, there appears to be a great deficiency in the use of an EB in their creation or the consultation of medical professionals in the content. In addition, rating the apps is variable, often reliant on user experience with a blunt 5 -star system and subjective written reviews of varying quality. When the Silberg scoring criteria were applied, the mean score was 3.26. Lack of universal and accepted accreditation is also a wider issue in healthcare technology.
Limitations of our study included potential reviewer error when documenting the app features, functionality and scoring. The authors tried to minimise this by using a two-author scoring system. The number of downloads was not accessible, thus the impact of each app on patients was unclear. Excluding apps that were too broad and may have included GORD as a part of their content is also a limitation, but the authors cannot be clear on the impact of this.

Further research in terms of systematic review of mHealth apps in general, as well as prospective and randomised trials, are needed to assess their impact. A specific scoring system to measure quality as well as regulating the development process to allow scientific rigour and ensure patient safety are needed. Stricter regulation should also come with an accreditation or certification process that is not voluntary.

\section{Acknowledgments}

Funding: This research did not receive any specific grant from funding agencies in the public, commercial, or notfor-profit sectors.

\section{Footnote}

Reporting Checklist: The authors have completed the PRISMA reporting checklist. Available at http://dx.doi. org/10.21037/mhealth-20-126

Conflicts of Interest: All authors have completed the ICMJE uniform disclosure form (available at http://dx.doi. org/10.21037/mhealth-20-126). The authors have no conflicts of interest to declare.

Ethical Statement: The authors are accountable for all aspects of the work in ensuring that questions related to the accuracy or integrity of any part of the work are appropriately investigated and resolved. Ethical approval was not required. All data was gathered from the public domain.

Open Access Statement: This is an Open Access article distributed in accordance with the Creative Commons Attribution-NonCommercial-NoDerivs 4.0 International License (CC BY-NC-ND 4.0), which permits the noncommercial replication and distribution of the article with the strict proviso that no changes or edits are made and the original work is properly cited (including links to both the formal publication through the relevant DOI and the license). See: https://creativecommons.org/licenses/by-nc-nd/4.0/. 


\section{References}

1. "Radio Telephone System". US Patent number 3,906,166; Filing date: 17 October 1973; Issue date: September 1975; Assignee Motorola.

2. Cell Phone Development - Motorola Solutions USA Motorola Solutions [Internet]. Motorolasolutions.com. Available online: https://www.motorolasolutions.com/en_ us/about/company-overview/history/explore-motorolaheritage/cell-phone-development.html

3. Number of smartphone users worldwide from 2016 to 2021(in billions) [Internet]. Statista 2020. Available online: https://www.statista.com/statistics/330695/number-ofsmartphone-users-worldwide/

4. Number of mobile phone users worldwide 2015-2020 [Internet]. Statista 2016. Available online: https://www. statista.com/statistics/274774/forecast-of-mobile-phoneusers-worldwide/

5. Mobile app usage - Statistics \& Facts [Internet]. Statista 2019. Available online: https://www.statista.com/ topics/1002/mobile-app-usage/

6. The Growing Value of Digital Health [Internet]. Iqvia 2017. Available online: https:/www.iqvia.com/insights/ the-iqvia-institute/reports/the-growing-value-of-digitalhealth

7. Jeon E, Park HA, Min YH, et al. Analysis of the information quality of korean obesity-management smartphone applications. Healthc Inform Res 2014;20:23-9.

8. Oh B, Yi GH, Han MK, et al. Importance of Active Participation in Obesity Management Through Mobile Health Care Programs: Substudy of a Randomized Controlled Trial. JMIR Mhealth Uhealth 2018;6:e2.

9. Stevens DJ, Jackson JA, Howes N, et al. Obesity surgery smartphone apps: a review. Obes Surg 2014;24:32-6.

10. O'Neill S, Brady RR. Colorectal smartphone apps: opportunities and risks. Colorectal Dis 2012;14:e530-4.

11. Carter T, O'Neill S, Johns N, et al. Contemporary vascular smartphone medical applications. Ann Vasc Surg 2013;27:804-9.

12. Mobasheri MH, Johnston M, King D, et al. Smartphone breast applications-what's the evidence? Breast 2014;23:683-9.

13. Connor K, Brady RR, de Beaux A, et al. Contemporary hernia smartphone applications (apps). Hernia 2014;18:557-61.

14. Xiao Q, Wang Y, Sun L, et al. Current Status and Quality Assessment of Cardiovascular Diseases Related
Smartphone Apps in China. Stud Health Technol Inform 2016;225:1030-1.

15. Abboudi H, Amin K. Smartphone applications for the urology trainee. BJU Int 2011;108:1371-3.

16. Hartz J, Yingling L, Powell-Wiley TM. Use of Mobile Health Technology in the Prevention and Management of Diabetes Mellitus. Curr Cardiol Rep 2016;18:130.

17. Morawski K, Ghazinouri R, Krumme A, et al. Association of a Smartphone Application With Medication Adherence and Blood Pressure Control: The MedISAFEBP Randomized Clinical Trial. JAMA Intern Med 2018;178:802-9.

18. Niendam TA, Tully LM, Iosif AM, et al. Enhancing early psychosis treatment using smartphone technology: A longitudinal feasibility and validity study. J Psychiatr Res 2018;96:239-46.

19. Cook KA, Modena BD, Simon RA. Improvement in Asthma Control Using a Minimally Burdensome and Proactive Smartphone Application. J Allergy Clin Immunol Pract 2016;4:730-7.e1.

20. Gastro-oesophageal reflux disease and dyspepsia in adults: investigation and management Guidance [Internet]. NICE 2014. Available online: https://www.nice.org.uk/guidance/ cg184/documents/dyspepsiagord-final-scope2

21. Evidence standards framework for digital health technologies [Internet]. NICE 2019. Available online: https://www.nice.org.uk/about/what-we-do/ourprogrammes/evidence-standards-framework-for-digitalhealth-technologies

22. Zhang MW, Ho RC, Hawa R, et al. Analysis of the Information Quality of Bariatric Surgery Smartphone Applications Using the Silberg Scale. Obes Surg 2016;26:163-8.

23. Buijink AW, Visser BJ, Marshall L. Medical apps for smartphones: lack of evidence undermines quality and safety. Evid Based Med 2013;18:90-2.

24. Najm A, Gossec L, Weill C, et al. Mobile Health Apps for Self-Management of Rheumatic and Musculoskeletal Diseases: Systematic Literature Review. JMIR Mhealth Uhealth 2019;7:e14730.

doi: 10.21037/mhealth-20-126

Cite this article as: Venugopal LS, Musbahi A, Shanmugam V, Gopinath B. A systematic review of smartphone apps for gastrooesophageal reflux disease: the need for regulation and medical professional involvement. mHealth 2021;7:56. 